\title{
Determinación experimental de la variación del coeficiente politrópico de compresión utilizando diagramas abiertos
}

Diego Christian Caputo, ${ }^{i}$ Rodolfo Oscar Berberi, ${ }^{i i}$ Néstor Alberto Emilio

Ferré, ,ii Fernando Gabriel Russo, ${ }^{\text {iv }}$ Gabriel Aram Receloglu, ${ }^{\vee}$ Pablo Gabriel

Cavataiovi \& Mauro José Brunovii

\section{Resumen}

El comportamiento del coeficiente politrópico durante la carrera de compresión en motores de combustión interna, define el área del ciclo indicado de la máquina. Cuando el ciclo se grafica en diagramas de presión - volumen, esta área representa el trabajo y también permite determinar la potencia.

El conocimiento de los valores que puede adoptar el coeficiente politrópico, así como la variación que experimenta durante la carrera de compresión, y su relación con el estado de carga del motor y el régimen de rotaciones, resulta de gran utilidad para la caracterización del fluido real de trabajo y la eficiencia energética de la máquina.

El presente trabajo muestra un sencillo procedimiento experimental que permite obtener una familia de curvas, a distintos regímenes de rotación del motor y a plena carga, que representan las variaciones del coeficiente politrópico a lo largo de la carrera de compresión. La obtención del diagrama abierto se realiza en un motor de automóvil, de cuatro tiempos y encendido a chispa, priorizando la sustitución del costoso equipamiento específico que habitualmente se utiliza en laboratorios de motores, por sensores industriales de uso corriente en la industria. Por su sencillez, el procedimiento puede ser emulado en cualquier laboratorio universitario de máquinas y motores, y permite obtener datos experimentales que sirven como insumo para el cálculo de nuevos desarrollos.

Palabras clave: coeficiente politrópico, diagrama indicado, potencia indicada, ciclo indicado, presión de compresión.

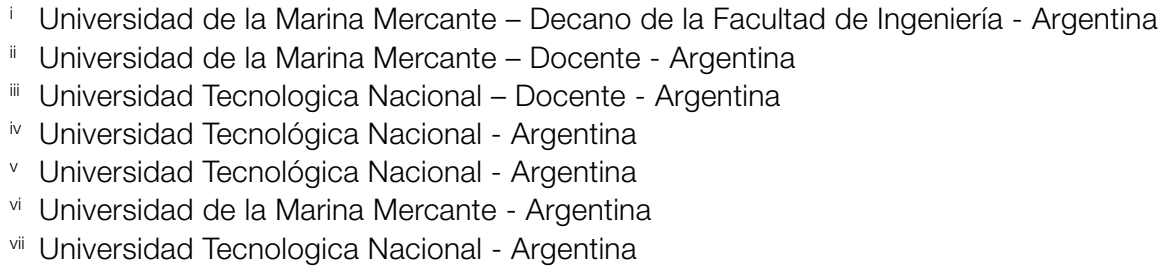




\section{Abstract}

The behavior of the polytropic coefficient during the compression stroke in internal combustion engines, defines the area of the indicated cycle of the machine. When the cycle is plotted in pressure - volume diagrams, this area represents the work and also allows to determinate power.

The knowledge of the values that the polytropic coefficient may adopt, as well as the variation experienced during the compression stroke, and its relation with the load conditions and rotation speed of the engine, is very useful for the characterization of the real working fluid and the energy efficiency of the machine.

This work presents a simple experimental procedure that allows to obtain a family of curves, at different rotating engine speeds and at full load, which represent the variations of the polytropic coefficient throughout the compression stroke. The acquire of the open diagram is done in a four stroke automobile engine with spark ignition, prioritizing the replacement of the expensive and specific equipment that is usually used in engine labs, by industrial sensors commonly used in the industry. Due to its simplicity, this procedure can be emulated in any machines and motors university laboratory, and allows to obtain experimental data that serve as input for the calculation of new developments.

Keywords: polytropic coefficient, indicated diagram, indicated power, indicated cycle, compression pressure.

Fecha de recepción 04/05/2017 - Fecha de aceptación 04/08/2017 


\section{Introducción}

En ingeniería, los modelos de cálculo, permiten abordar situaciones complejas realizando una serie de simplificaciones. Sin restar esencia, estas prácticas resuelven problemas de la ingeniería, en tanto su encuadre corresponda a las condiciones de borde acotadas para la aplicación del modelo. Existen diversos tipos de modelos que pueden aplicarse a una situación real: los modelos numéricos presentan correlaciones basadas en series matemáticas cuyos coeficientes se pueden calcular con la ayuda de software especializado, pero también existen modelos basados en la determinación experimental de coeficientes, que aplicados a formulaciones teóricas permiten aproximar resultados que verifican el comportamiento real de un fenómeno.

El ciclo indicado previsto CIPREV, es una herramienta para modelar los ciclos de trabajo de los motores de combustión interna. Bajo esta concepción, el ciclo se obtiene realizando una serie de pruebas de carácter complementario, las cuales pueden componerse con posterioridad como si se tratara de una aplicación del concepto de superposición de efectos. En estas pruebas se busca determinar valores de parámetros que caractericen la evolución del fluido de trabajo del motor, como por ejemplo el coeficiente politrópico en la fase compresión, los calores específicos a presión y volumen constante del fluido, y su constante particular asociada a considerarlo un gas ideal, entre otras.

Cada vez que el método se aplica a la constatación de motores existentes, las pruebas proporcionan los datos para el cálculo y verificación del caso particular; pero también engrosan el acervo de datos por los cuales los valores que adoptan las variables descriptas se transforman en datos de diseño para nuevos motores.

En el presente trabajo se describe el procedimiento experimental de un conjunto de pruebas que conforman la aplicación práctica del modelo CIPREV para la verificación de motores existentes. En esta etapa del proyecto de investigación se ha sustituido el costoso equipamiento específico por sensores de uso corriente en la industria, de modo que el método puede ser emulado en cualquier laboratorio universitario de motores con fines de experimentación y didácticos.

\section{Modelo empleado}

El área del ciclo constituye el trabajo que se obtiene del fluido. El mismo opera entre los volúmenes fijos $\mathrm{V}_{1}$, volumen total del cilindro, $\mathrm{y}_{2} \mathrm{o}$ volumen de la cámara de combustión. Éstos se verifican cuando el pistón se encuentra respectivamente en el PMI y PMS. Siendo fijos $\mathrm{V}_{1} \mathrm{y}_{2}$, las curvas politrópicas de compresión y expansión definen el área de trabajo. En la figura 1 se muestra el ciclo teórico del motor en estudio donde, entre los puntos 1 y 2 del diagrama, se grafica la transformación politrópica de compresión, y entre 3 y 4, la de expansión. 


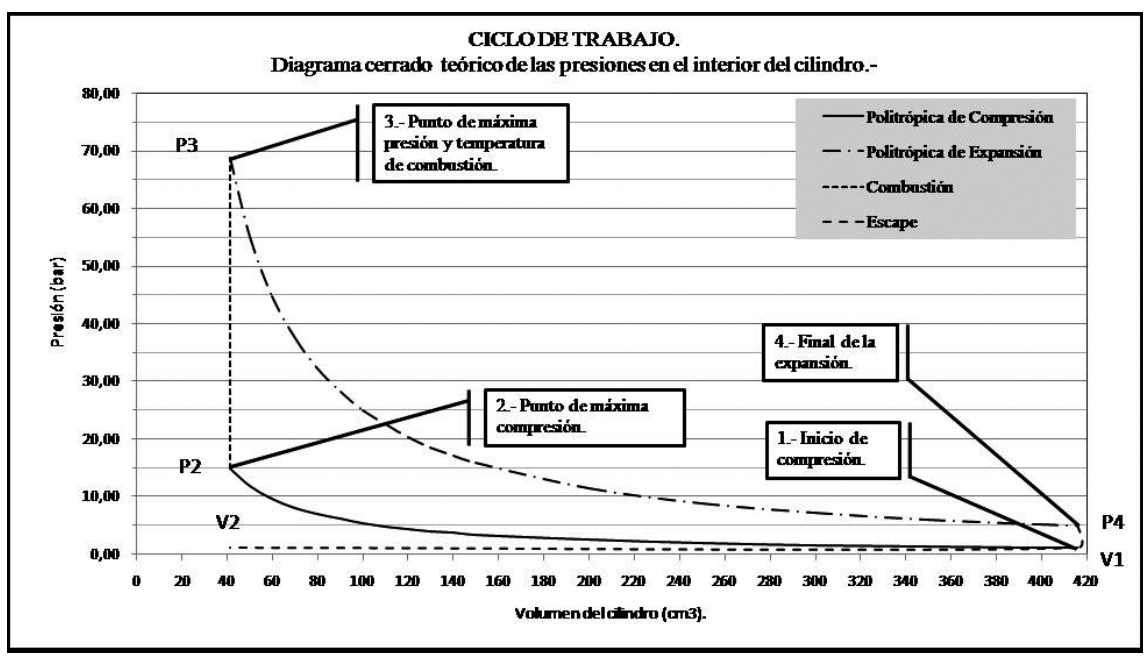

Figura 1: diagrama $\mathrm{P}$ - V teórico cuya área representa el trabajo en el cilindro.

En la figura 2 se ha substituido en el eje de abscisas el volumen del cilindro por los grados de giro correspondiente a la manivela del eje del motor. Obteniéndose así, el diagrama abierto de las presiones dentro del cilindro. En línea llena puede apreciarse el ciclo sin combustión (que es el que se determinará mediante el proceso experimental descripto en este trabajo) y en línea de trazos la influencia del proceso de combustión sobre el desarrollo de la presión interior del cilindro.

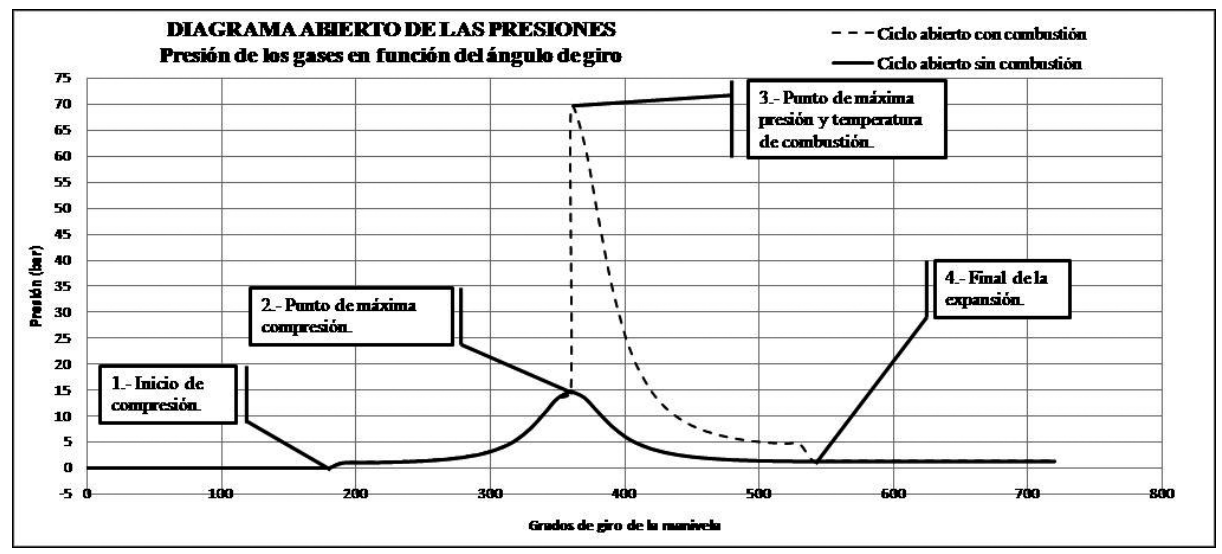

Figura 2: diagrama abierto de las presiones en el cilindro.

Durante la carrera de compresión la máquina realiza trabajo sobre el fluido, esto ocurre entre los puntos 1 y 2 del diagrama de la figura 1 . En el motor real esta transformación es de tipo politrópica y obedece a la formulación siguiente: 


$$
p_{1} \cdot V_{1}^{n}=p_{2} \cdot V_{2}^{n}
$$

Donde $\mathrm{n}$ es el coeficiente politrópico que responde al grado de permeabilidad a los intercambios de calor del sistema y varía a lo largo de la carrera. Durante la expansión ocurre un fenómeno similar que puede formularse reemplazando en la (1) $\mathrm{p}_{1}$ por $\mathrm{p}_{4}$ y $\mathrm{p}_{2}$ por $\mathrm{p}_{3}$, de acuerdo a lo que se desprende del análisis de las figuras 1 y 2 .

Llamando relación de compresión $\varepsilon$ al cociente entre el volumen máximo del cilindro $\mathrm{V}_{1}$ y el volumen de la cámara de combustión $\mathrm{V}_{2}$. Y asumiendo que existen infinitas relaciones que definen el grado de reducción del volumen dentro del cilindro a lo largo de la carrera del pistón, puede definirse una relación $\varepsilon^{\prime}$ que vincule al volumen inicial $\mathrm{V}_{1}$ con un volumen genérico cualquiera $\mathrm{V}_{\mathrm{x}}$. Donde $\mathrm{V}_{\mathrm{x}}$ representa el volumen que se observa en el interior del cilindro en un punto cualquiera de la carrera.

$$
\varepsilon^{\prime}=\frac{V_{1}}{V_{x}}
$$

Combinando la ecuación (1) y (2) puede obtenerse la ecuación general que describe la variación de presión en el interior del cilindro durante la carrera de compresión:

$$
P_{y}=P_{1}\left(\frac{V_{1}}{V_{x}}\right)^{n}
$$

En el dominio de la función (3) deberá cumplirse que $V_{2} \leq V_{x} \leq V_{1}$, y en consecuencia, a dichos valores de Vx le corresponderán valores de Py que cumplirán con la condición $P_{2} \geq P_{y} \geq P_{1}$.

El coeficiente politrópico que define la transformación de compresión puede obtenerse partiendo de la ecuación (3):

$$
n=\frac{\log \left(\frac{P_{y}}{P_{1}}\right)}{\log \left(\frac{V_{1}}{V_{x}}\right)}
$$

El volumen $\mathrm{V}_{\mathrm{x}}$ observado en el interior del cilindro cuando el pistón se desplaza producto del corrimiento angular de la manivela puede calcularse en función de dicho ángulo, teniendo en cuenta la geometría del sistema biela manivela:

$$
V_{x}=S \cdot\left[r \cdot(1-\cos \alpha)+\frac{r}{\lambda} \cdot\left(1-\sqrt{1-\lambda^{2} \operatorname{sen}^{2} \alpha}\right)\right]+V_{2}
$$

Donde $\mathrm{S}$ es la superficie del cilindro, $\alpha$ el ángulo recorrido por la manivela 
respecto del PMS, $r$ es el radio de la manivela y $\lambda=r / L$, relación de volteo.

Idénticamente combinando (1) y (2) aplicadas a la carrera de expansión se pueden obtener las ecuaciones que permiten establecer la variación de presión durante dicha carrera, y el coeficiente politrópico que la caracteriza:

$$
\begin{gathered}
P_{y}=P_{4}\left(\frac{V_{1}}{V_{x}}\right)^{n} \\
n=\frac{\log \left(\frac{P y}{P_{4}}\right)}{\log \left(\frac{v_{1}}{V_{x}}\right)}
\end{gathered}
$$

El método para el cálculo del trabajo consiste en encontrar el área por debajo de la politrópica de compresión (trabajo de compresión que la máquina ejerce sobre el fluido). El área por debajo de la curva de compresión estará dada por la integral de la función (3), calculada entre los volúmenes V1 y V2. En su forma general puede escribirse como:

$$
T_{c}=\int_{V_{2}}^{V_{1}} P_{y} d v
$$

Sustituyendo Py por su valor dado por (3) y resolviendo la integral, la expresión final del trabajo de compresión queda expresada como sigue:

$$
T_{c}=P_{1} \cdot V_{1}^{n} \cdot\left(\frac{V_{1}^{-n+1}-V_{2}^{-n+1}}{-n+1}\right)
$$

Análogamente se puede proceder para determinar el trabajo de la expansión Te y por diferencia con el de compresión obtener el trabajo indicado Ti desarrollado por el ciclo.

$$
\begin{gathered}
T_{e}=P_{4} \cdot V_{1}^{n} \cdot\left(\frac{V_{1}^{-n+1}-V_{2}^{-n+1}}{-n+1}\right) \\
T_{i}=\left(P_{4}-P_{1}\right) \cdot V_{1}^{n} \cdot\left(\frac{V_{1}^{-n+1}-V_{2}^{-n+1}}{-n+1}\right)
\end{gathered}
$$

Finalmente, para obtener la potencia indicada Pi en función de la presión media indicada pmi del ciclo, puede recurrirse a los métodos tradicionales difundidos ampliamente en la literatura especializada, por ejemplo de la siguiente forma:

$$
\begin{gathered}
p m i=\frac{T_{i}}{V_{c}} \\
P_{i}=\frac{p m i \cdot V_{c t} . N}{900}
\end{gathered}
$$


Donde $\mathrm{Vc}$ es el volumen de cilindrada unitario y Vct el total del motor, y $\mathrm{N}$ el número de rotaciones por minuto. En el denominador, la constante 900 permite obtener la potencia en $\mathrm{CV}$, cuando $\mathrm{Vct}$ es expresado en $\mathrm{dm}^{3}$, pmi en $\mathrm{kg} / \mathrm{cm} 2$ y el ciclo es de cuatro tiempos.

\section{Desarrollo experimental}

El desarrollo experimental tiene por objetivo hallar el diagrama abierto de las presiones, sin fase de combustión, en el cilindro $\mathrm{n}^{\circ} 4$ de un motor de encendido a chispa.

El mismo se lleva a cabo en cuatro etapas. En la primera se buscará ubicar el punto muerto superior PMS e instalar el sensor de referencia angular. En la segunda, con el motor a temperatura de régimen, se extraerá la bujía de encendido del cilindro número 4, y se instalará un sensor de presión industrial. Se hará funcionar el motor de combustión en tres cilindros a diferentes rotaciones y a plena carga, obteniendo los diagramas superpuestos de variación de presión en el cilindro de prueba, sin fase de combustión, y las referencias angulares del PMS. En la tercera etapa, se realizarán pruebas de banco a plena carga con medición de los productos de combustión y consumo específico. Esto tiene por finalidad calcular los parámetros de presión y temperatura desarrollados durante el proceso de combustión.

En la etapa final se realizará un ensayo convencional de potencia al freno, obteniendo la curva de potencia efectiva del motor. De esta forma se podrán calcular los rendimientos y los parámetros energéticos del motor, tomando como ciclo indicado, el modelado con los datos aportados durante el ensayo de ciclo abierto, y como parámetros efectivos los del ensayo final de potencia al freno.

Como sensor de referencia angular, se ha escogido un sensor de la marca Siemens, serie Simatic PXI 200, modelo 3RG4075-0AJ00 con frecuencia de operación hasta $100 \mathrm{~Hz}$.

Para la medición de la presión desarrollada en el interior del cilindro se optó por un sensor industrial marca Danfoss, modelo MBS 3000, con una presión máxima de trabajo de 16 bar, una temperatura máxima de operación de $85^{\circ} \mathrm{C}$. y velocidad de respuesta $<4 \mathrm{~ms}$.

El equipamiento de medición se completa con un osciloscopio digital marca GW Instek GDS - 2062 y una memoria de almacenamiento digital USB portátil.

\subsection{Determinación del PMS e instalación del sensor de referencia angular}

Para la determinación exacta del PMS se instala un disco graduado en la polea del cigüeñal y un puntero laser como referencia fija externa al motor. Se rosca en el orificio de la bujía de encendido un tope mecánico que interrumpe la carrera 
del pistón cuando se gira en un sentido arbitrario cualquiera. Hecho tope, se gira en sentido contrario midiendo el corrimiento angular con el puntero laser sobre el disco graduado hasta que el pistón vuelve a hacer contacto contra el tope mecánico. En estas condiciones, el ángulo que falta girar en el mismo sentido para alcanzar el PMS se calcula como sigue:

$$
\text { ángulo al } P M S=\frac{\left(360^{\circ} \text {-angulo medido }\right)}{2}
$$

En la figura 3 se aprecia el disco graduado ya montado sobre la polea del cigüeñal y el puntero laser midiendo el recorrido angular sobre el mismo.

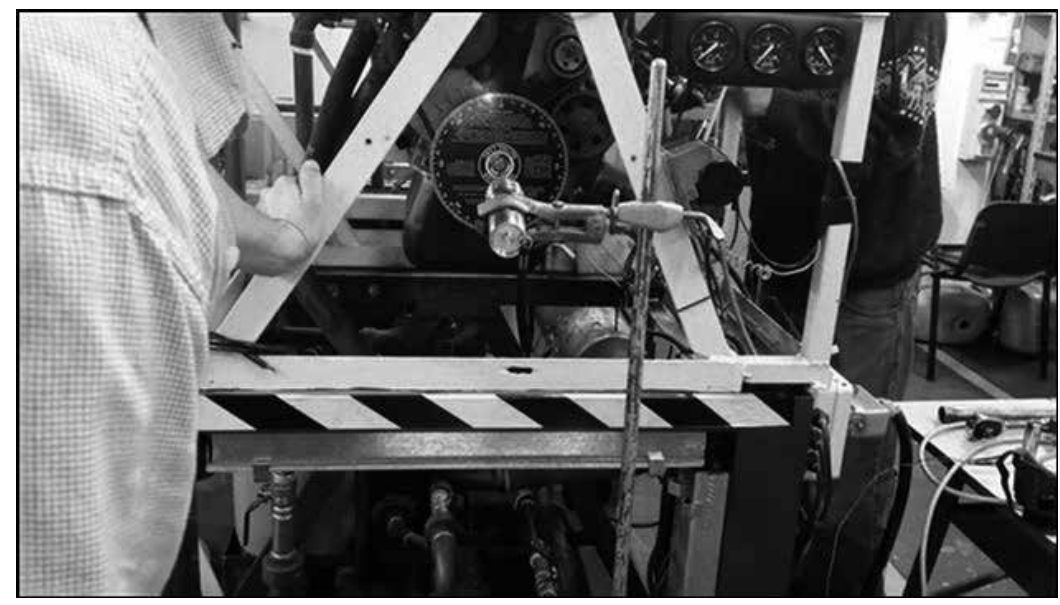

Figura 3: disco graduado instalado sobre la polea del cigüeñal y puntero laser midiendo la posición angular del PMS para fijación del sensor.

\subsection{Determinación del ciclo abierto de las presiones.}

Una vez que el motor ha llegado a temperatura de régimen, se extrae la bujía de encendido correspondiente al cilindro número 4 y se instala el sensor de presión industrial. Situación que se muestra en la figura 4. 


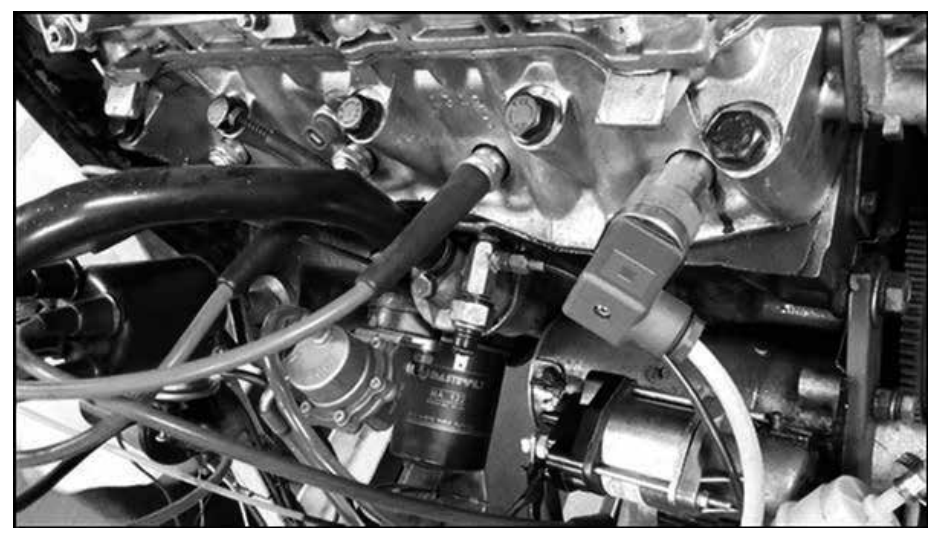

Figura 4: sensor de presión Danfoss MBS 3000 instalado en el orificio de la bujía de encendido del cilindro $n^{\circ} 4$.

Se enciende el motor nuevamente funcionando en tres cilindros. Se aplica carga con el freno dinamométrico hidráulico hasta alcanzar el régimen de plena carga manteniendo constante las rotaciones. Alcanzada esta condición de funcionamiento, se procede a la captura de datos con el osciloscopio digital. La misma consiste en obtener la forma de onda correspondiente a la caída de tensión en la resistencia de carga RL, que resulta proporcional a la presión desarrollada. En la figura 5 se muestra como se conectan ambos canales del osciloscopio para registrar las tensiones al mismo tiempo.

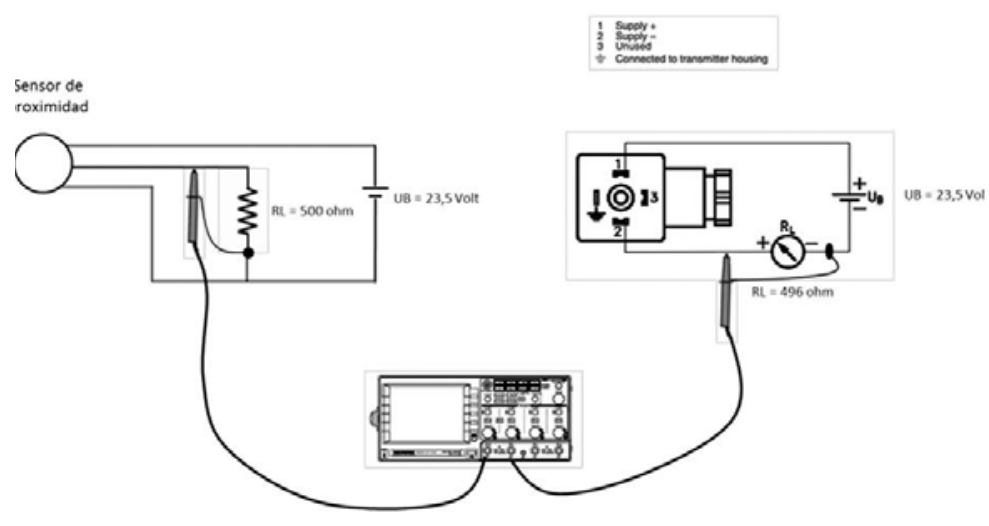

Figura 5: esquema de conexiones del osciloscopio digital para la toma de los datos de presión y referencia angular.

Para pasar los resultados en tensión a valores de presión se ha realizado la calibración previa del sensor de presión. Del proceso de calibración se obtiene la ecuación lineal que permite la conversión directa, donde P simboliza la presión 
medida y $\mathrm{V}_{\mathrm{RL}}$ la caída de tensión en la resistencia de carga del sensor de presión:

$$
P=\frac{V_{R L}-2}{0,5}
$$

3.3. Medición de los productos de combustión y ensayo de potencia al freno.

Para la aplicación del modelo presentado, resulta imprescindible completarlo hallando el trabajo y la potencia, para compararlos con los datos obtenidos de pruebas tradicionales de potencia al freno y calcular los rendimientos. Para ello se realizará una prueba de banco adicional a plena carga, con el objeto de obtener datos que permitan calcular la entalpía de los gases y su temperatura teórica durante el proceso de combustión. Luego aplicando el modelo de los gases ideales se calculará la presión desarrollada durante la combustión.

\section{Resultados}

Para este estudio se realizaron ensayos a 1100, 2200, 3300 y 4100 r.p.m. todos a plena carga con el motor funcionando en tres cilindros, mientras se registraba la presión y posición del PMS en el cilindro número 4.

Las figuras 6, 7, 8 y 9 muestran la captura de imagen del osciloscopio midiendo las señales de los sensores de referencia angular (señal pulsante cuadrada) y de presión (señal ondulante) para los ensayos citados. Ambas señales se observan en valores de tensión correspondiente a la caída en las resistencias de carga y en función del tiempo.

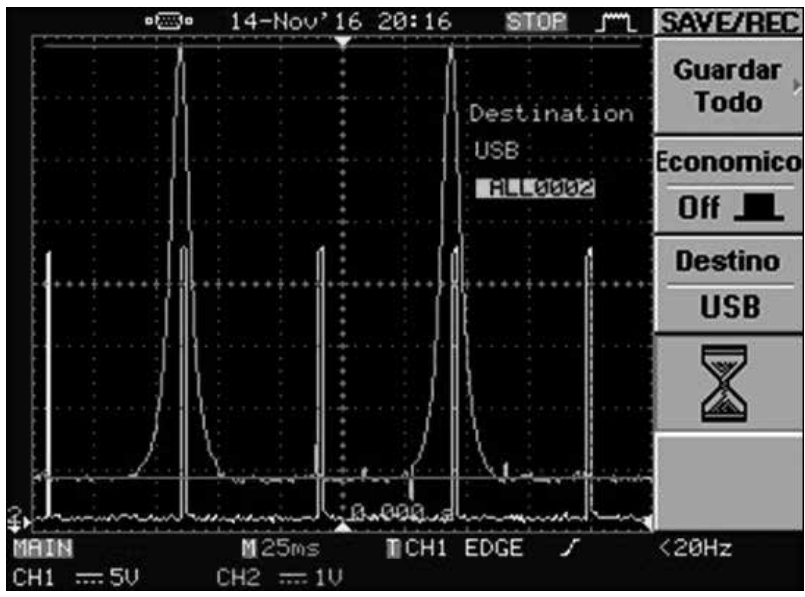

Figura 6: captura del osciloscopio a 1100 r.p.m. El canal $1(\mathrm{CH} 1)$ corresponde a la señal de PMS y el canal $2(\mathrm{CH} 2)$ a la señal de presión. 


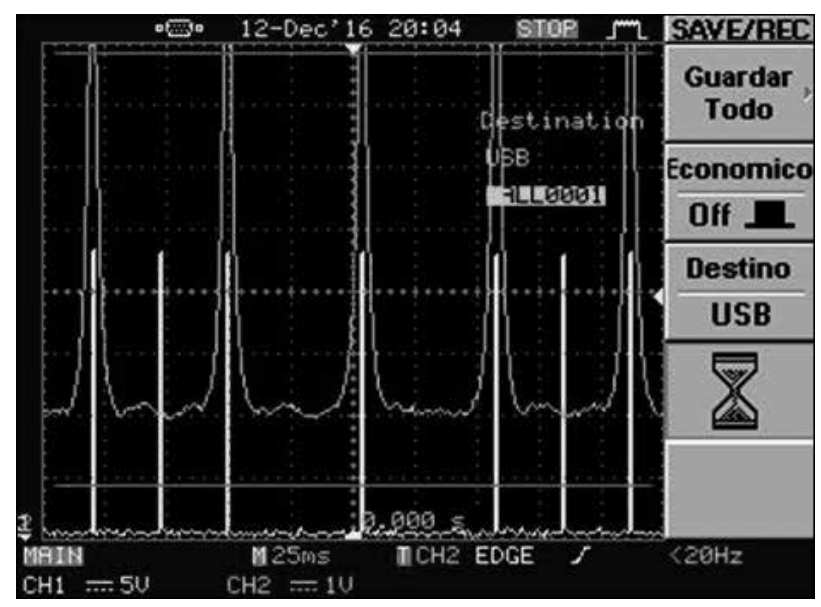

Figura 7: captura del osciloscopio a 2200 r.p.m. El canal $1(\mathrm{CH} 1)$ corresponde a la señal de PMS y el canal $2(\mathrm{CH} 2)$ a la señal de presión.

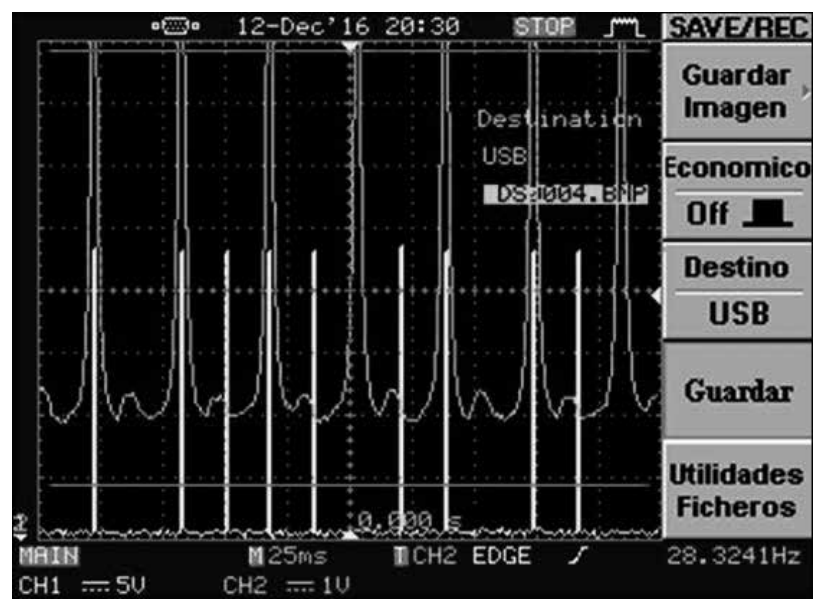

Figura 8: captura del osciloscopio a 3300 r.p.m. El canal $1(\mathrm{CH} 1)$ corresponde a la señal de PMS y el canal $2(\mathrm{CH} 2)$ a la señal de presión. 


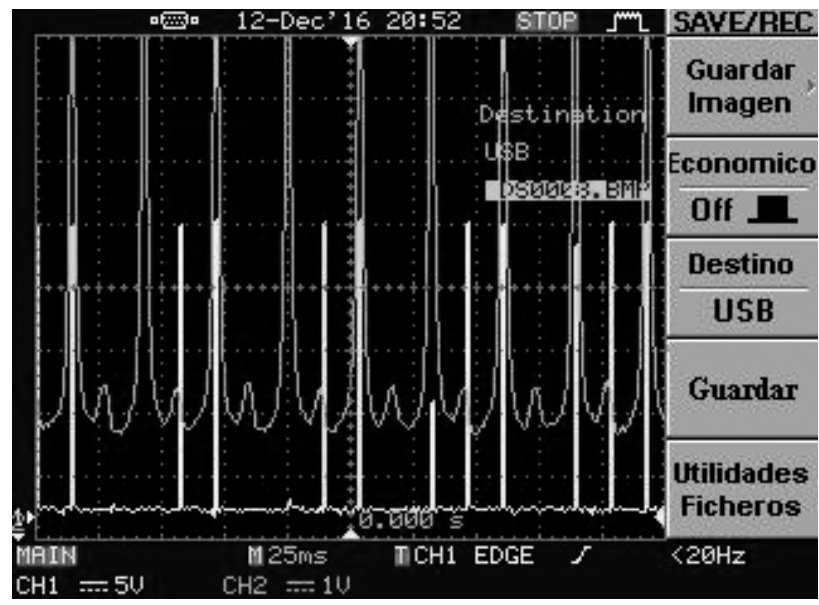

Figura 9: captura del osciloscopio a 4100 r.p.m. El canal $1(\mathrm{CH} 1)$ corresponde a la señal de PMS y el canal $2(\mathrm{CH} 2)$ a la señal de presión.

Para el análisis matemático de la señal, se ha pasado la captura de imagen del osciloscopio a planilla de cálculo, y aplicando la formula (16) se han convertido los valores de tensión a valores de presión. El paso siguiente consiste en dividir cada ensayo por ciclos de motor completos (determinados por los pulsos del sensor de PMS) y hallar el ciclo abierto de las presiones más representativo de cada prueba. Esto puede observarse en la figura 10, en la que la base de tiempo ha sido reemplazada por los grados de giro de la manivela del eje cigüeñal.

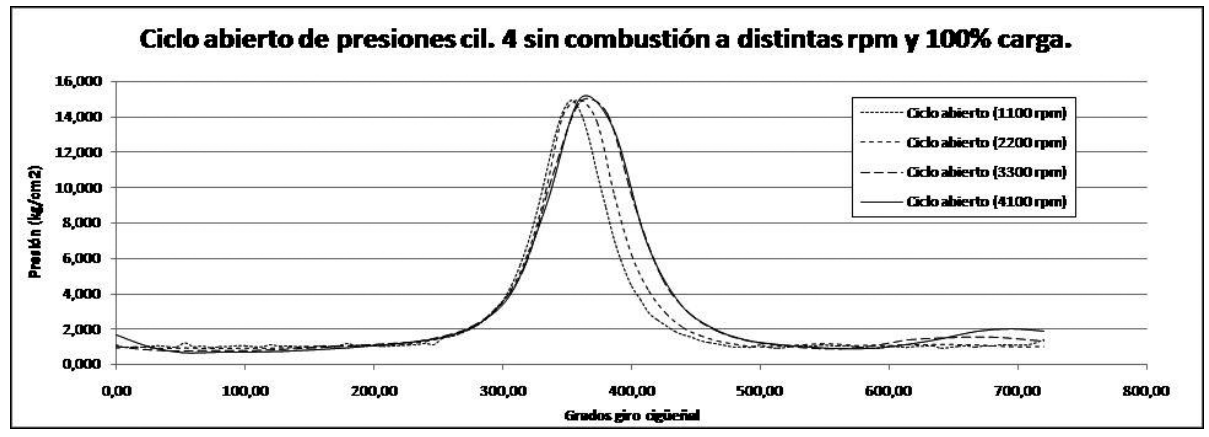

Figura 10: ciclo abierto sin combustión a distintas r.p.m. y a plena carga.

Por último, segmentando por carrera el diagrama abierto, y sustituyendo en abscisas los grados de giro de la manivela por el volumen del cilindro utilizando la ecuación (5), se puede obtener el detalle de la evolución de la presión en la fase de compresión.

En las figuras 11, 12, 13 y 14 se observa la presión desarrollada durante la carrera de compresión real, y también se puede observar la curva que describe 
el coeficiente politrópico. En superposición con la curva de presión medida se ha trazado la curva de presión calculada con el modelo CIPREV, utilizando el coeficiente politrópico medio.

Para el cálculo del coeficiente politrópico medio $n$, el intervalo considerado es toda la carrera, ya que para la aplicación de la fórmula (4) se toman los volúmenes extremos $\mathrm{V}_{1} \mathrm{y} \mathrm{V}_{2} \mathrm{y}$ las presiones medidas que corresponden a dichos volúmenes. Los valores del coeficiente politrópico medio se informan en cada figura, entre paréntesis, en el título de la misma, junto al dato de las rotaciones a las que se tomaron los valore de presión.

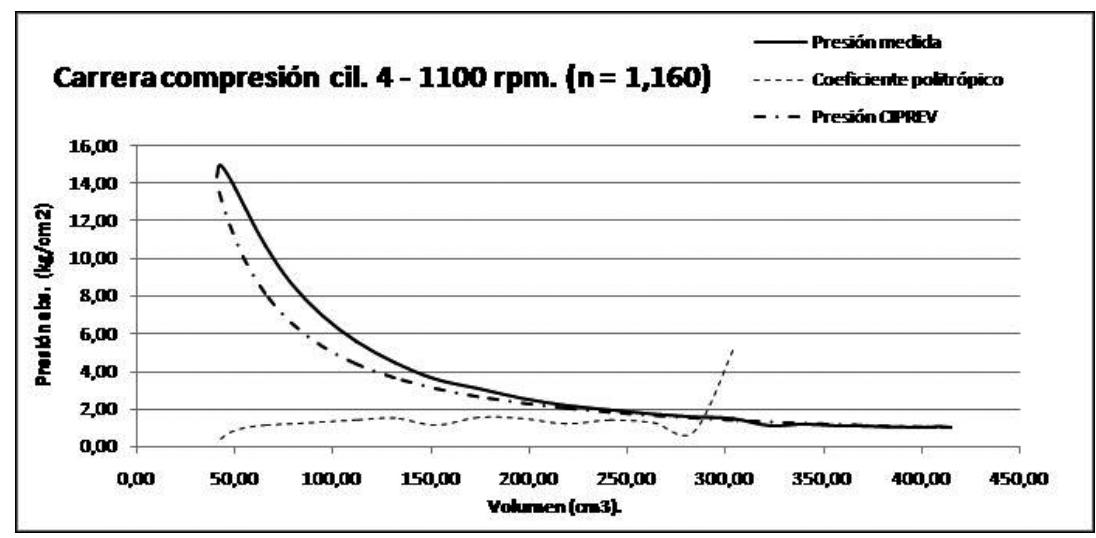

Figura 11: presión durante la compresión a 1100 r.p.m., comparación con la presión modelada, y variación del coeficiente politrópico.

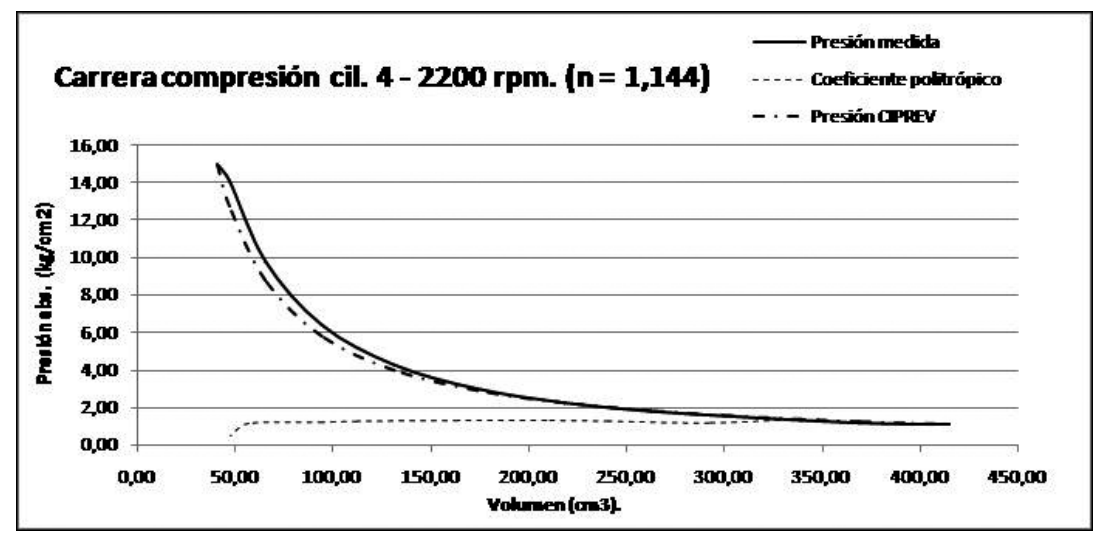

Figura 12: presión durante la compresión a 2200 r.p.m., comparación con la presión modelada, y variación del coeficiente politrópico. 


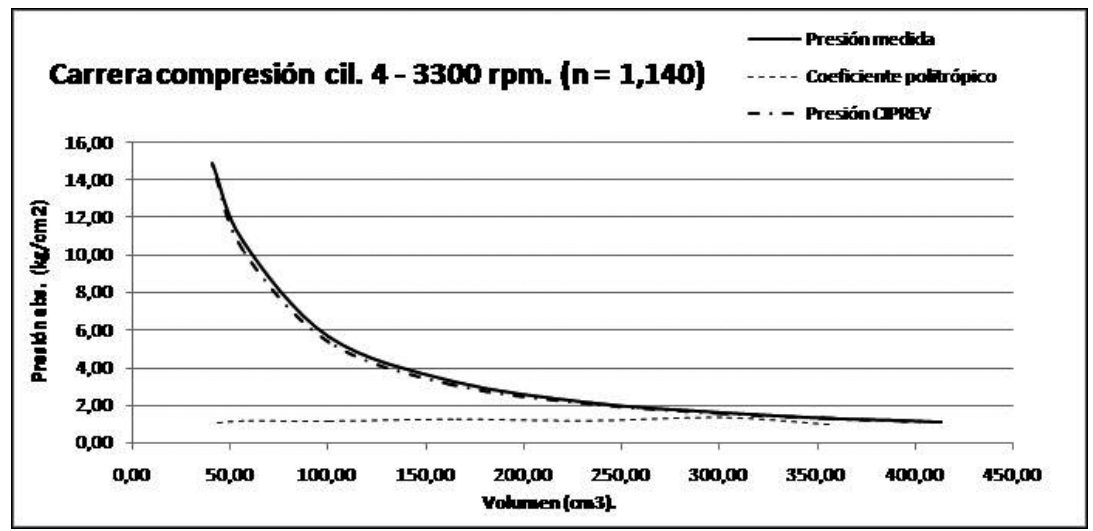

Figura 13: presión durante la compresión a 3300 r.p.m., comparación con la presión modelada, y variación del coeficiente politrópico.

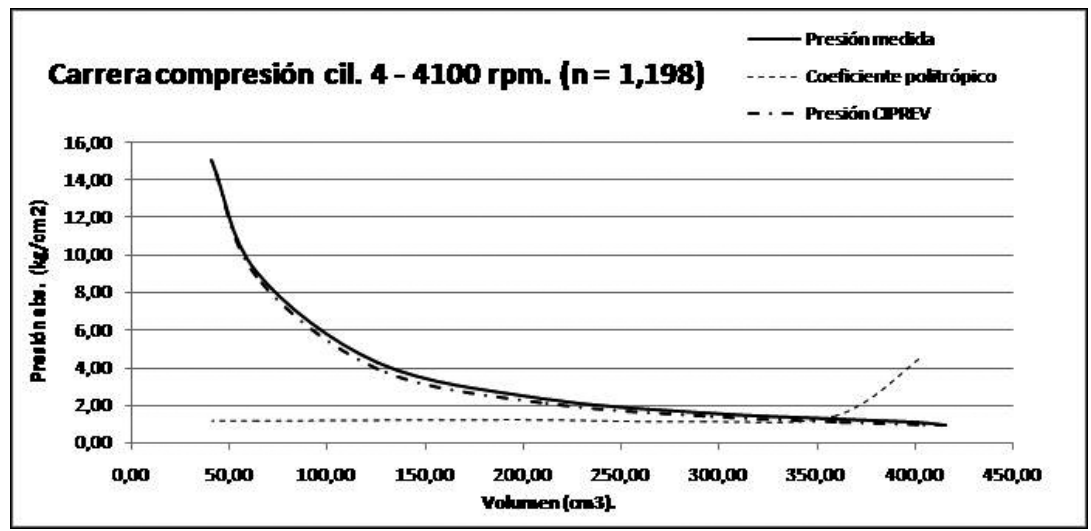

Figura 14: presión durante la compresión a 4100 r.p.m., comparación con la presión modelada, y variación del coeficiente politrópico.

El análisis de gases de combustión se realizó en una prueba separada y sus resultados se muestran en la tabla 1 . 
Tabla 1: resultado del ensayo de potencia al freno con medición de los productos de combustión y consumo específico.

\begin{tabular}{|c|c|c|c|c|}
\hline \multicolumn{5}{|c|}{ 4.2.- MEDICIÓN DE GASES DE COMBUSTIÓN } \\
\hline \multirow{2}{*}{ Código } & \multirow{2}{*}{ EP 003} & \multirow{2}{*}{$\mathrm{N}^{\circ}$ ensayo } & \multirow{2}{*}{ EP 003-003/15 } & Fecha: \\
\hline & & & & $11 / 08 / 2015$ \\
\hline Duración & $9,5 \mathrm{~min}$ & Laboratorio & \multicolumn{2}{|c|}{ Termofluidos.- F.I.- UdeMM. } \\
\hline \multicolumn{5}{|c|}{ 4.2.1.- CONDICIONES DE ENSAYO.- } \\
\hline \multicolumn{2}{|c|}{ Vel. De rotación (RPM) } & 2116,87 & \multirow{2}{*}{\multicolumn{2}{|c|}{$\begin{array}{c}\text { Caract. Teórica del } \\
\text { combustible }\end{array}$}} \\
\hline \multicolumn{2}{|c|}{ Fuerza (kg) } & 18,84 & & \\
\hline \multicolumn{2}{|c|}{ Potencia (CV) } & 39,88 & \multicolumn{2}{|c|}{$\mathrm{C} 8 \mathrm{H} 18$} \\
\hline \multicolumn{2}{|c|}{ Par Motor (kgm) } & 13,49 & \multicolumn{2}{|c|}{ Masa molecular } \\
\hline \multicolumn{2}{|c|}{ Cons. Especifico (kg/CV.h) } & 0,1628 & \multicolumn{2}{|c|}{$114 \mathrm{~kg} / \mathrm{kmol}$} \\
\hline \multicolumn{2}{|c|}{ Cons. p/ciclo de trabajo $(\mathrm{kg})$} & $1,02226 \times 10^{4}$ & \multicolumn{2}{|c|}{ Aire estequeométrico } \\
\hline \multicolumn{2}{|c|}{ Pc teórico del comb. (kcal/kg) } & $10.526,30$ & \multicolumn{2}{|c|}{$15,25 \mathrm{~kg} / \mathrm{kg}$ combustible. } \\
\hline \multicolumn{5}{|c|}{ 4.2.2.- RESULTADOS EXPERIMENTALES.- } \\
\hline \multicolumn{4}{|c|}{ Variable medida } & \multirow[b]{2}{*}{ Valor medido } \\
\hline orden & Magnitud & $\begin{array}{c}\text { Abreviatura/ } \\
\text { Símbolo }\end{array}$ & Unidad & \\
\hline 1 & Oxigeno libre & $\mathrm{O} 2$ & $\%$ & 1 \\
\hline 2 & \begin{tabular}{|l|}
$\begin{array}{l}\text { Monóxido } \\
\text { Carbono }\end{array}$ \\
\end{tabular} & $\mathrm{CO}$ & ppm & 4297 \\
\hline 3 & Exceso de aire & $\lambda$ & -n-n-n & 1,05 \\
\hline 4 & \begin{tabular}{|l|l|}
$\begin{array}{l}\text { Dióxido de } \\
\text { Carbono }\end{array}$ \\
\end{tabular} & $\mathrm{CO} 2$ & $\%$ & 14,76 \\
\hline 5 & Pérdida por humos & $\mathrm{pA}$ & $\%$ & 14,7 \\
\hline 6 & Tiro & $* * * * * * * *$ & $\mathrm{hPa}$ & $* * * * * * *$ \\
\hline 7 & Temp. Ambiente & TA & ${ }^{\circ} \mathrm{C}$ & 21,1 \\
\hline 8 & \begin{tabular}{|l|} 
Temp. de los \\
productos de \\
Combustión
\end{tabular} & TH & ${ }^{\circ} \mathrm{C}$ & 386,4 \\
\hline 9 & \begin{tabular}{|l|} 
Rendimiento de la \\
combustión
\end{tabular} & Ren & $\%$ & 85,2 \\
\hline \multirow[b]{2}{*}{15} & \multicolumn{4}{|c|}{ OBSERVACIONES } \\
\hline & $\begin{array}{l}\text { Carburación: } A \\
\text { boca }=200, \mathrm{Al}\end{array}$ & $\begin{array}{l}\text { Aire } 1^{\circ} \text { boca }= \\
\text { ta } 2^{\circ} \text { boca }=102\end{array}$ & $\begin{array}{l}\text { 220, Alta } 1^{\circ} \text { boca } \\
\text { 2.- }\end{array}$ & $=110 /$ Aire $2^{\circ}$ \\
\hline \multicolumn{5}{|c|}{ 4.2.3.- INSTRUMENTO UTILIZADO.- } \\
\hline \multirow{3}{*}{16} & \multirow{3}{*}{$\begin{array}{c}\text { Equipo } \\
\text { Utilizado }\end{array}$} & Marca & \multicolumn{2}{|l|}{ Testo } \\
\hline & & \begin{tabular}{|l|} 
Modelo \\
\end{tabular} & \multicolumn{2}{|l|}{ T 310} \\
\hline & & $\mathrm{n}^{\circ}$ serie & \multicolumn{2}{|l|}{42818294} \\
\hline
\end{tabular}

En la sección 4.2.1 de la tabla 1 se observan los resultados obtenidos con el freno dinamométrico hidráulico, y datos del combustible considerado para la aplicación del modelo. En la sección 4.2.2 la composición de los gases de combustión obtenida con el analizador de gases Testo 310 durante dicha prueba.

El cálculo estequiométrico muestra que la masa total de fluido que ingresa al cilindro para tomar parte del proceso de combustión en cada ciclo operativo equivale a $17,01 \mathrm{~kg} / \mathrm{kg}$ de combustible. 
Las mismas reglas estequiométricas permiten determinar la cantidad total de gases producto de la combustión en volumen Gt; esto se muestra en la tabla 2. En la columna 10 se puede apreciar que la cantidad de productos de combustión en masa (kg de producto por $\mathrm{kg}$ de combustible) es igual a la de los productos ingresantes, lo que verifica el balance de masas. Para obtener una información detallada sobre el proceso de cálculo de los productos de combustión se puede consultar [1] Caputo, D y otros.

Tabla 2: cantidad de moles por producto, y total de la mezcla de gases de combustión.

\begin{tabular}{|c|c|c|c|c|c|c|c|c|c|c|}
\hline \multicolumn{11}{|c|}{ PRODUCTOS DE COMBUSTIÓN } \\
\hline 1 & 2 & 3 & 4 & 5 & 6 & 7 & 8 & 9 & 10 & 11 \\
\hline \multirow{4}{*}{$\begin{array}{c}\text { Productos de } \\
\text { la } \\
\text { combustión } \\
\text { propuestos. }\end{array}$} & \begin{tabular}{|c|} 
Gases totales \\
de \\
combustión \\
en volumen
\end{tabular} & $\begin{array}{c}\% \text { en } \\
\text { volúmen }\end{array}$ & Presión & Volúmen & $\begin{array}{c}\text { Constante } \\
\text { universal }\end{array}$ & $\begin{array}{c}\text { Temperat } \\
\text { ura }\end{array}$ & $\begin{array}{c}\text { Número de } \\
\text { moles }\end{array}$ & $\begin{array}{c}\text { Masa } \\
\text { molecular }\end{array}$ & $\begin{array}{c}\text { masa } \\
\text { productos de } \\
\text { la combustión }\end{array}$ & $\begin{array}{c}\% \text { en masa } \\
\text { prod. } \\
\text { Combustión }\end{array}$ \\
\hline & Gtv & \begin{tabular}{|c|}
$\%$ \\
volumen
\end{tabular} & $\mathbf{P}$ & v & $\mathrm{R}$ & T & $\mathrm{n} 1$ & M1 & $\mathrm{m} 1$ & $\%$ masa \\
\hline & calculado & medido & convención & \begin{tabular}{|l|} 
calculado \\
\end{tabular} & dato & medido & calculado & dato & calculado & calculado \\
\hline & ( Nm3/kg comb) & (\%) & $(\mathrm{kg} / \mathrm{m} 2)$ & $\begin{array}{c}\text { (Nm3/kg } \\
\text { comb) }\end{array}$ & $\begin{array}{c}\text { ikgf.m/kmol.K } \\
1 \\
\end{array}$ & $(\mathrm{~K})$ & $\begin{array}{c}(\mathrm{kmol} / \mathrm{kg} \\
\mathrm{comb})\end{array}$ & $(\mathrm{kg} / \mathrm{kmol})$ & (kg/kg comb) & (x) \\
\hline $\mathrm{O}_{2}$ & \multirow{7}{*}{13,121} & 1 & 10000 & 0,1312 & 848 & 659,55 & 0,00235 & 32 & 0,0751 & 0,441 \\
\hline $\mathrm{CO}_{2}$ & & 14,76 & 10000 & 1,9367 & 848 & 659,55 & 0,03463 & 44,01 & 1,5239 & 8,959 \\
\hline $\mathrm{CO}$ & & 0,4297 & 10000 & 0,0564 & 848 & 659,55 & 0,00101 & 28,01 & 0,0282 & 0,166 \\
\hline $\mathrm{N}_{2}$ & & & 10000 & & 848 & 659,55 & 0,38247 & 28,016 & 10,7152 & 62,992 \\
\hline $\mathrm{H}_{2} \mathrm{O}$ & & & 10000 & & 848 & 659,55 & 0,04006 & 18,016 & 0,7218 & 4,243 \\
\hline NO & & & 10000 & & 848 & 659,55 & 0,11513 & 30 & 3,4540 & 20,305 \\
\hline $\begin{array}{c}\mathrm{HC} \text { (como } \\
\mathrm{C}_{8} \mathrm{H}_{18} \text { no } \\
\text { quemado) }\end{array}$ & & & 10000 & & 848 & 659,55 & 0,04098 & 12,01 & 0,4921 & 2,893 \\
\hline TOTALES & & & & & & & 0,61662 & & 17,0104 & 100 \\
\hline
\end{tabular}

La entalpía de los gases de combustión $\mathrm{h}_{\mathrm{c}}$ puede obtenerse como el cociente entre el poder calorífico del combustible Pc, y los gases totales de combustión Gt. Para que el valor de la entalpía de los gases represente el caso particular, se afectará el cociente por el rendimiento de la combustión registrado para este ensayo por analizador de gases:

$$
h_{c}=\frac{P_{c}, \eta_{c}}{G t}
$$

Para el caso en estudio, el valor de hc $=684 \mathrm{kcal} / \mathrm{Nm} 3$, dicho valor permite hallar la temperatura de combustión con la ayuda del diagrama de Rosin - Fehling para combustiones con muy bajo exceso de aire. Diagrama cuya adaptación, para este trabajo, se muestra en la figura 15; y que permite inferir que la combustión se desarrolló con una temperatura aproximada de $1700^{\circ} \mathbf{C}$. 


\section{Curva de Rosin - Fehling \\ Humos de escape - Hidrocarburos con Pci mayor a $10000 \mathrm{kCal} / \mathrm{kg} "$ )}

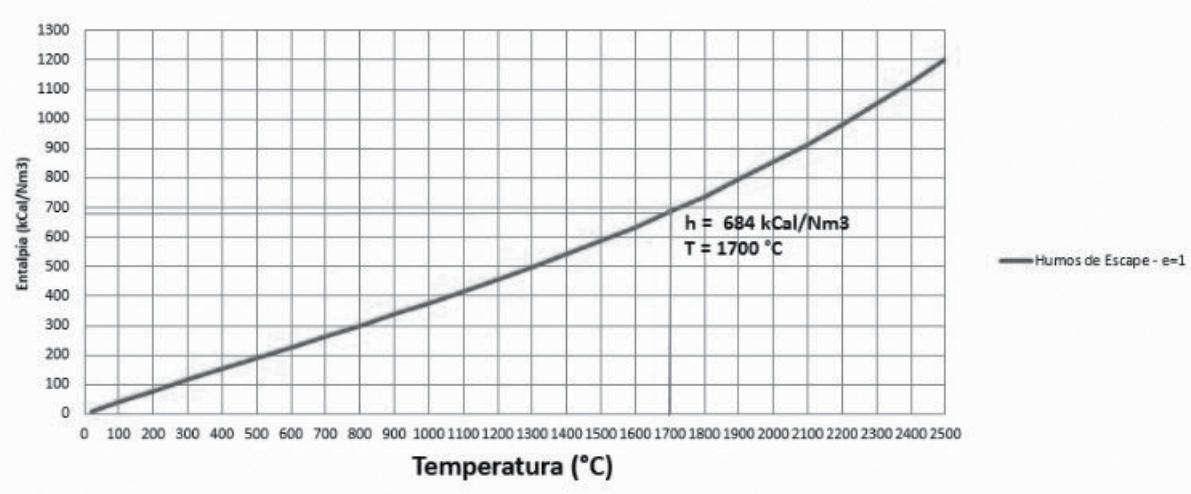

Figura 15: diagrama Rosing - Fehling para combustiones con bajo exceso de aire.

Por aplicación de la ley de los gases ideales a las transformaciones a volumen constante, se determina la máxima presión de combustión $\mathrm{P}_{3}$.

La tabla 3 muestra los valores de presión y temperatura para los puntos característicos del diagrama de trabajo presión - volumen, que a modo de ejemplo, se presentó en la figura 1. Los mismos se presentan en forma ordenada para los ensayos de ciclo abierto, realizados a 1100, 2200, 3300 y 4100 r.p.m. respectivamente.

Tabla 3: parámetros del ciclo indicado previsto CIPREV, calculados con los datos del ensayo de ciclo abierto a distintos regímenes de velocidad y a plena carga.

\begin{tabular}{|c|c|c|c|c|c|c|c|c|c|c|}
\hline \multirow{4}{*}{\multicolumn{2}{|c|}{\begin{tabular}{|c|} 
\\
Aplicación del modelo \\
del ciclo previsto: \\
ensayo preliminar. \\
Valor de P2 obtenido \\
\end{tabular}}} & \multicolumn{9}{|c|}{ CALCULO DEL CICLO PREVISTO PARA EL CILINDRO N ${ }^{\circ} 4$} \\
\hline & & 1 & 2 & 3 & 4 & 5 & 6 & 7 & 8 & 9 \\
\hline & & $\begin{array}{l}\text { Presión de } \\
\text { admisión }\end{array}$ & $\begin{array}{l}\text { Temperatura } \\
\text { inicio } \\
\text { compresión }\end{array}$ & $\begin{array}{c}\text { Exponente } \\
\text { politrópico de } \\
\text { compresión }\end{array}$ & $\begin{array}{c}\text { Presión final } \\
\text { de } \\
\text { compresión }\end{array}$ & $\begin{array}{l}\text { Temperatura } \\
\text { final de } \\
\text { compresión }\end{array}$ & $\begin{array}{l}\text { Presión final } \\
\text { de } \\
\text { combustión }\end{array}$ & $\begin{array}{c}\text { Temperatura } \\
\text { de combustión }\end{array}$ & $\begin{array}{l}\text { Presión } \\
\text { apertura } \\
\text { válvulas } \\
\text { escape }\end{array}$ & $\begin{array}{l}\text { Exponente } \\
\text { politrópico } \\
\text { de expansión }\end{array}$ \\
\hline & & P1 & $\mathrm{T} 1$ & n & P2 & $\mathrm{T} 2$ & P3 & T3 & P4 & n \\
\hline & & (bar) & $\left({ }^{\circ} \mathrm{C}\right)$ & & (bar) & $\left({ }^{\circ} \mathrm{C}\right)$ & (bar) & $\left({ }^{\circ} \mathrm{C}\right)$ & (bar) & \\
\hline & & \multirow{2}{*}{ medido } & \multirow{2}{*}{$\begin{array}{c}\text { medido } \\
\text { c/camara } \\
\text { termográfica }\end{array}$} & calculado & \multirow{2}{*}{ medido } & calculado & calculado & calculado & calculado & calculado \\
\hline $\begin{array}{l}\text { Marca/ } \\
\text { Modelo }\end{array}$ & $\begin{array}{c}\text { Cilindro } \\
\mathrm{N}^{*}\end{array}$ & & & formula (4) & & $\mathrm{T} 2=\mathrm{T} 1 \cdot \varepsilon^{\wedge}(\mathrm{n}-1)$ & $\begin{array}{c}\mathrm{P} 3= \\
\mathrm{P} 2(\mathrm{~T} 3 / \mathrm{T} 2)\end{array}$ & $\begin{array}{c}\text { Grafico Rosin - } \\
\text { Felhing }\end{array}$ & $\mathrm{P} 4=\mathrm{P} 3 / \varepsilon^{\mathrm{n}}$ & formula (7) \\
\hline \multirow{4}{*}{$\begin{array}{c}\text { Fiat Regatta } \\
85-1500 \\
\text { c.c. }\end{array}$} & 4 & 0,98 & 72 & 1,159 & 14,32 & 225,66 & 56,65 & 1700,00 & 4,65 & 1,159 \\
\hline & 4 & 1,06 & 72 & 1,143 & 14,93 & 207,65 & 61,27 & 1700,00 & 5,20 & 1,143 \\
\hline & 4 & 1,07 & 72 & 1,137 & 14,84 & 200,29 & 61,85 & 1700,00 & 5,33 & 1,137 \\
\hline & 4 & 1,07 & 72 & 1,142 & 15,02 & 206,03 & 61,85 & 1700,00 & 5,27 & 1,142 \\
\hline
\end{tabular}


Para verificar la aplicabilidad del modelo, se realiza un ensayo de banco convencional con el objeto de obtener la curva completa de potencia efectiva. Esta curva suministrará los datos experimentales que permiten evaluar el desempeño energético del motor, a la vez que su análisis, permitirá inferir las fortalezas y debilidades del método empleado para calcular el ciclo indicado.

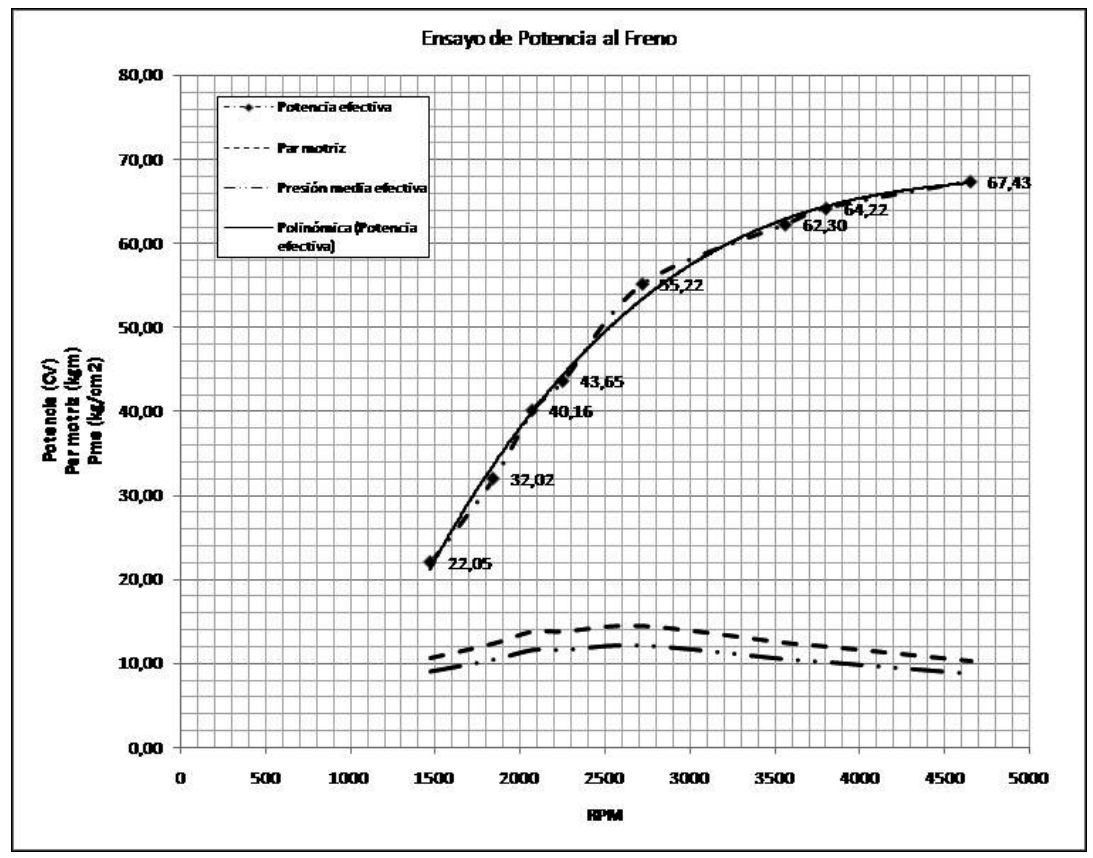

Figura 16: curva de potencia efectiva obtenida durante un ensayo de banco convencional.

Con los datos de los puntos característicos del ciclo de trabajo calculados para el cilindro $\mathrm{n}^{\circ} 4$ del motor, se calculan el trabajo indicado y la potencia. Estos valores se comparan con los resultados de la potencia efectiva al mismo número de rotaciones, y de esa comparación surgen los rendimientos térmico y mecánico de la máquina. 
Tabla 2: parámetros energéticos y rendimiento del ciclo modelado.

\begin{tabular}{|c|c|c|c|c|c|c|c|c|}
\hline \multicolumn{9}{|c|}{ PARAMETREOS ENERGÉTICOS DEL CICLO CON EL MODELO CIPREV. } \\
\hline 10 & 11 & 12 & 13 & 14 & 15 & 16 & 17 & 18 \\
\hline $\begin{array}{l}\text { Trabajo de } \\
\text { compresión }\end{array}$ & $\begin{array}{l}\text { Trabajo de } \\
\text { expansión }\end{array}$ & \begin{tabular}{|c|} 
Trabajo \\
indicado del \\
ciclo
\end{tabular} & $\begin{array}{l}\text { Velocidad } \\
\text { angular }\end{array}$ & $\begin{array}{l}\text { Presión } \\
\text { media del } \\
\text { ciclo }\end{array}$ & $\begin{array}{l}\text { Rendimiento } \\
\text { Térmico }\end{array}$ & $\begin{array}{l}\text { Potencia } \\
\text { Indicada } \\
\text { (total del } \\
\text { motor) }\end{array}$ & $\begin{array}{l}\text { Potencia } \\
\text { efectiva } \\
\text { (total del } \\
\text { motor) }\end{array}$ & $\begin{array}{c}\text { Rend. } \\
\text { Mecánico }\end{array}$ \\
\hline Tc & $\mathrm{Te}$ & Li & $\mathrm{N}$ & pm & nt & Pi & $\mathrm{Pe}$ & $\mathrm{nm}$ \\
\hline$(\mathrm{kgm})$ & $(\mathrm{kgm})$ & $(\mathrm{kgm})$ & $(1 / \min )$ & (bar) & $(\%)$ & (CV) & (CV) & (\%) \\
\hline calculado & calculado & calculado & \multirow[b]{2}{*}{ medido } & Calculado & calculado & calculado & \multirow[b]{2}{*}{ medido } & Calculado \\
\hline formula (10) & formula (11) & formula (12) & & $\mathrm{pmi}=\mathrm{L} / \mathrm{Vc}$ & $\mathrm{nt}=1-(\mathrm{T} 1 / \mathrm{T} 2)$ & $\begin{array}{c}\mathrm{Pi}= \\
\text { (Li.N.n }{ }^{\circ} \text { cil)/ } \\
9000\end{array}$ & & $\eta \mathrm{m}=\mathrm{Pe} / \mathrm{Pi}$ \\
\hline 11,40 & 54,06 & 42,67 & 1100 & 11,39 & 0,31 & 20,86 & 10,00 & $47,94 \%$ \\
\hline 12,09 & 59,35 & 47,26 & 2200 & 12,61 & 0,28 & 46,21 & 43,00 & $93,05 \%$ \\
\hline 12,10 & 60,29 & 48,18 & 3300 & 12,86 & 0,27 & 70,67 & 61,00 & $86,32 \%$ \\
\hline 12,18 & 59,99 & 47,81 & 4100 & 12,76 & 0,28 & 87,12 & 66,00 & $75,76 \%$ \\
\hline
\end{tabular}

\section{Discusión}

El ciclo abierto de las presiones en el interior del cilindro, sin fase de combustión, resulta un método fácil de aplicar y poco costoso para determinar el coeficiente politrópico durante la carrera de compresión.

El coeficiente politrópico de compresión decrece a medida que la carrera de compresión progresa y disminuye el volumen en el interior del cilindro. Esta variación es más acentuada a bajas vueltas que a altas rotaciones.

El coeficiente politrópico medio, que se calcula teniendo en cuanta la máxima presión $\mathrm{P}_{2}$ desarrollada durante el intervalo correspondiente a toda la carrera de compresión, parece representar con bastante fidelidad el comportamiento del coeficiente real a rotaciones cercanas a las máximo par motor. Puede verse claramente en las figuras 11 a 14, como la curva de presión modelada con el exponente politrópico medio, se aproxima a la curva de presión medida. Y cómo también, la variación del coeficiente real, es cada vez menor aproximándose a una pendiente casi nula a altas rotaciones, en concordancia con el coeficiente medio.

En relación al modelo del ciclo indicado previsto CIPREV, los valores de trabajo, potencia y rendimiento guardan relación con los usualmente aceptados por la literatura especializada en la temática, lo que motiva la profundización del estudio para hacer extensiva la aplicación del modelo a otros tipos de motores térmicos. 


\section{Referencias}

D. Caputo, R. Berberi, N. Ferré, A. Puricelli, V. Fonteriz, R. Ferreira, R. Aguirre, B. Calvo y J. Bruno, "El análisis de los productos de combustión y su uso en el cálculo de la máxima presión de combustión en motores de ciclo Otto", en Actas III CADI 2016.

D. Giacosa, Motores Endotérmicos, Barcelona: Editorial Omega, 1998.

A. C. García, Termodinámica Técnica, Buenos Aires: Editorial Alsina, 2006.

A. J. Torregosa y P. Olmeda, "Perdidas de calor. Refrigeración", en Motores de Combustión Interna Alternativos, Barcelona: Editorial Reverté, 2014.

D. Barilá, L. Bugna, F. Vignolo, R. Gómez, P. Kolodka y G. Ahrtz, "Obtención del diagrama indicado en motores de combustión interna sin necesidad de referencia angular", en Actas I CAIM 2008.

F. Soto Pau, J. A. Silva, A. M. Dos Santos, "Cálculo de la temperatura en el interior de la cámara de combustión en motores de combustión interna", en Ingeniería Mecánica, vol. 5, núm. 2, pp 7-15, 2002.

M. Lapuerta, "Study of de compression cycle of reciprocating engine through the polytropic coefficient". Elsevier Science Ltd. Valencia (2002).

M. Thiem, Termotécnia, Manual del Ingeniero Técnico, vol. XX, Bilbao: Editorial Urmo, 1974. 